\title{
Recent progress in autophagy
}

Cell Research (2014) 24:1-2. doi:10.1038/cr.2014.1; published online 2 January 2014

Autophagy is a lysosome-based degradation process whose primary function is to degrade long-lived proteins and recycle cellular components. So far, 3 types of autophagy have been documented, including macroautophagy, microautophagy and chaperone-mediated autophagy. Autophagy has been shown to degrade cargo in selective and noselective manners. In literature, the term "autophagy" usually indicates macroautophagy. Macroautophagy is characterized by the formation of double membrane vesicles termed autophagosomes. The biogenesis of autophagosomes is a subject of intensive study in recent years. Autophagy can be induced by various stimuli, including nutrient starvation, hypoxia and various chemical reagents such as rapamycin. In yeast, the major physiological function of autophagy is to adjust the metabolic machinery in response to varying nutrient status, thereby ensuring the cell survival in an ever-changing environment. In mammalian cells, however, the roles of autophagy are more complex. Autophagy has been implicated in many different cellular processes as varied as cell survival, death, pathogen clearance, and antigen presentation. Various diseases have been linked to dysfunctioning autophagy. There are strong genetic and animal data supporting the direct connections between autophagy and cancers, neurodegenerative diseases, and chronic inflammation. Albeit the exact mechanisms of autophagy in each of the diseases remain to be determined, modulating autophagy has become a target for therapeutic approaches for various diseases.

During the early days after its discovery in 1950 s, autophagy had been considered as a relatively backwater field. Identification of genes regulating autophagy in 1990s opened up the field. In the past two decades, autophagy study has grown explosively. As a result, our understanding in every aspect of autophagy has been dramatically expanded. In this special issue, the history of autophagy study, the molecular machinery of autophagy, how nutrient signaling regulates autophagy, the biogenesis of autophagosome, the role of autophagy in various diseases, autophagy study in the model organism $C$. elegans, mitophagy, and chaperone-mediated autophagy (CMA) are reviewed and summarized by leading experts in the autophagy field.

Shortly after the discovery of lysosomes by Christian de Duve in 1950s, autophagosome was observed using electron microscopy. In 1963, de Duve coined the term autophagy, which referred to the delivery of intracellular contents to lysosomes. In the next 30 years, although some progress had been made, autophagy largely remained to be an obscure field due to the lack of understanding at the molecular level. The turning point of autophagy research came at early 1990s when Yoshinori Ohsumi discovered that autophagy could also happen in yeast. This discovery led Ohsumi and other groups to discover a set of "ATG" genes, which opened the possibility to understand the molecular and cellular mechanisms of autophagy and to dissect the roles of autophagy in physiological and pathological settings.

The leading piece of this special issue is a review about the historical landmarks of autophagy research, authored by Yoshinori Ohsumi. Dr
Ohsumi summarizes the key discoveries of autophagy research, with an emphasis on the earlier phase of the field and a particular focus on studies carried out in yeast. Immediately after the identification of "ATG" genes, various groups started to dissect the roles of these ATG genes in autophagy. Tremendous progress has been made in the past two decades in terms of understanding the molecular mechanism of autophagy, the molecular machinery that governs various steps of autophagy, and the structures of some of the key molecules involved. Dr Klionsky and colleagues summarize these advances in their review article. Nutrient starvation is the most widely used and arguably the most physiologically relevant stimulus of autophagy. Cell senses nutrient starvation by nutrient-sensitive kinases such as $\mathrm{mTORC} 1$ and AMPK. Through these kinases, signaling is passed to the autophagy machinery by various autophagy-specific signaling complexes including the Atg1/ULK1 complex and Vps34 complex. Extensive crosstalk and feedback loops have been discovered recently, highlighting the complex and dynamic nature of the autophagy signaling pathway. In their review article, Dr Guan and colleagues summarize the current understanding of how nutrient signaling regulates autophagy. The biogenesis of autophagosomes involves sophisticated and extensive membrane reshuffling. Various intracellular membrane systems including the ER, Golgi, mitochondrion, plasma membrane, and endocytic vesicles have been implicated in autophagosome formation. Despite the complex nature of autophagosome biogenesis, an increasingly clear picture of autophagosome formation is 
emerging from recent progress in the field. Some of the long standing key questions on autophagosome formation, including the membrane source, and where and how autophagsosomes are made, have been addressed or at least partially addressed. These advances in our understanding of autophagosome formation are summarized in the review article by Drs Shibutani and Yoshimori. Although autophagy has been shown to play import roles in various physiological settings and autophagydeficient animals develop phenotypes closely similar to human diseases, the direct link between dysfunction or misregulation of autophagy and human diseases has only been reported fairly recently. Various clinical trials of autophagy- modulating reagents have been carried out. The review by Drs Jiang and Mizushima summarizes the recent progress on this important front. Model organisms have been used extensively in autophagy study. Besides the yeast and mammalian systems, C. elegans has emerged as an important model system to study autophagy, especially for selective autophagy and the role of autophagy in developmental settings. The current knowledge of autophagy in C. elegans is summarized in the review by Drs Yang and Zhang. Besides macroautophagy, there are other types of autophagy. CMA is a pathway in which CMA substrate proteins are selectively targeted into lysosomes for degradation. CMA selectively degrades proteins in a timely fashion, thus playing important roles in regulating various physiological processes. Drs Cuervo and Wong summarize our current understanding of CMA. Cargos including organelles, protein aggregates, and intracellular pathogens can be recognized and engulfed by selective autophagy. Mitophagy, which degrades damaged mitochondria, is the most studied form of selective autophagy. A lot of progress has been made recently in understanding the molecular mechanism and physiological relevance of mitophagy, which is covered in a review by Dr Okamoto and colleagues in a later issue.
So far, there are many well written reviews on the subject of autophagy. Nevertheless, due to the rapid progress of autophagy research, new discoveries need to be summarized and synthesized in a timely fashion. Therefore, a timely update about the latest discoveries is certainly useful for "insiders" of the autophagy field. On the other hand, more and more new researchers have been drawn into this fascinating field, and thus a collection of reviews which cover the major topics in autophagy are especially useful for those who have just joined the autophagy community. We hope that the collection of authoritative reviews in this special issue on autophagy will prove useful for all researchers in this fast growing field.

\section{$\mathrm{Li} \mathrm{Yu}^{1}$}

${ }^{I}$ Center for Life Sciences, School of Life Sciences, Tsinghua University, Beijing 100084, China

liyulab@mail.tsinghua.edu.cn 